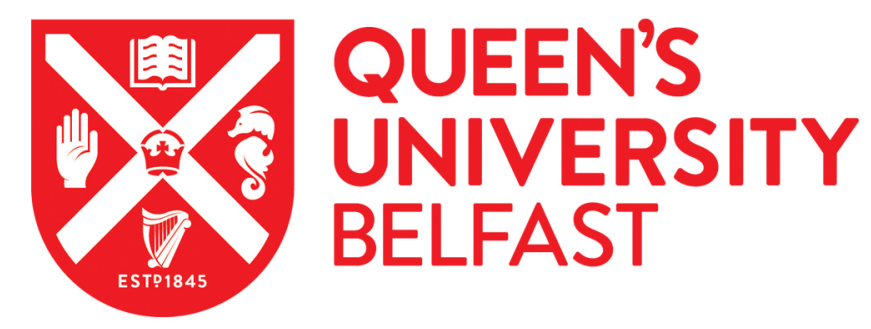

\title{
Experimental signature of quantum Darwinism in photonic cluster states
}

Ciampini, M. A., Pinna, G., Mataloni, P., \& Paternostro, M. (2018). Experimental signature of quantum Darwinism in photonic cluster states. Physical Review A, 98(2), [020101].

https://doi.org/10.1103/PhysRevA.98.020101

Published in:

Physical Review A

Document Version:

Publisher's PDF, also known as Version of record

Queen's University Belfast - Research Portal:

Link to publication record in Queen's University Belfast Research Portal

\section{Publisher rights}

(c) 2018 American Physical Society. This work is made available online in accordance with the publisher's policies. Please refer to any applicable terms of use of the publisher.

\section{General rights}

Copyright for the publications made accessible via the Queen's University Belfast Research Portal is retained by the author(s) and / or other copyright owners and it is a condition of accessing these publications that users recognise and abide by the legal requirements associated with these rights.

Take down policy

The Research Portal is Queen's institutional repository that provides access to Queen's research output. Every effort has been made to ensure that content in the Research Portal does not infringe any person's rights, or applicable UK laws. If you discover content in the Research Portal that you believe breaches copyright or violates any law, please contact openaccess@qub.ac.uk. 


\title{
Experimental signature of quantum Darwinism in photonic cluster states
}

\author{
Mario A. Ciampini, ${ }^{1,2,{ }^{*}}$ Giorgia Pinna, ${ }^{1}$ Paolo Mataloni, ${ }^{1}$ and Mauro Paternostro ${ }^{3}$ \\ ${ }^{1}$ Dipartimento di Fisica, Sapienza Università di Roma, P.le Aldo Moro 5, 00185 Rome, Italy \\ ${ }^{2}$ University of Vienna, Faculty of Physics, Vienna Center for Quantum Science and Technology (VCQ), \\ Boltzmanngasse 5, 1090 Vienna, Austria \\ ${ }^{3}$ Centre for Theoretical Atomic, Molecular and Optical Physics, School of Mathematics and Physics, \\ Queen's University Belfast, Belfast BT7 1NN, United Kingdom
}

(Received 20 March 2018; published 2 August 2018)

\begin{abstract}
We report on an experimental assessment of the emergence of Quantum Darwinism (QD) from engineered open-system dynamics. We use a photonic hyperentangled source of graph states to address the effects that correlations among the elements of a multiparty environment have on the establishment of objective reality ensuing the quantum-to-classical transition. Besides embodying one of the first experimental efforts toward the characterization of QD, our work illustrates the nontrivial consequences that multipartite entanglement and, in turn, the possibility of having environment-to-system back-action have on the features of the QD framework.
\end{abstract}

DOI: 10.1103/PhysRevA.98.020101

Introduction. The field of quantum open systems attempts at shedding light on the process translating the nonclassical state of a system to a mundane classical description [1-3]. The origin of such a mechanism-which is dubbed quantum-toclassical transition - and the features of its occurrence remain not completely clear or understood, and are the topic of much research effort [4,5]. One of the most accredited explanations relies on the action of environment-induced decoherence [6], a phenomenon where the environment surrounding a given system continuously monitors the state of the latter, thus acquiring information about it. The consequence of the environmental monitoring process is that fragile quantum superpositions of the system under scrutiny are removed in time, while classical mixtures of more robust macroscopic states survive, leading to the transition to classicality [7].

Often, in light of the typical assumption of an environment consisting of a very large number of subsystems whose dynamics is virtually impossible to track, it is more convenient to keep the focus on the system and its properties. The environment is thus eliminated from the dynamics, and the effect of its coupling to the system retained "effectively" in the properties of the ensuing system's nonunitary evolution. However, much can be learned from keeping the state of the environment and shifting the attention to the features of the informationacquisition process at the basis of environment-induced decoherence. This is precisely what Quantum Darwinism (QD) aims at doing [8].

Consider a quantum system $\mathcal{S}$ interacting with an environment $\mathcal{E}$ made out of many independent (and noninteracting) subsystems $\mathcal{E}^{(j)}$. External observers wanting to get information about $\mathcal{S}$ are allowed to do so only by directly measuring $\mathcal{E}_{j}$. The environment is thus a witness to the system's state: the information that the former gains over the latter is used to learn about the state of $\mathcal{S}$. Although, at first sight, this might sound

\footnotetext{
"marioarnolfo.ciampini@uniroma1.it
}

surprising, such indirect inference process is fully consistent with the theory of quantum measurement: a system is learnt only by measuring a fraction of the environment it interacts with (such as, for instance, the detection apparatus).

In the QD picture the classical description of $\mathcal{S}$ emerges from the quantum one due to the proliferation of redundant information throughout the environment resulting from $\mathcal{S}-\mathcal{E}$ correlations. More precisely, key to QD is that the states produced by environment-induced decoherence encode many local copies of classical information about $\mathcal{S}$. Such a proliferation allows information about $\mathcal{S}$ to be extracted from different fragments of the environment, and intercepted by observers.

The larger the number of fragments that acquire information $\sim S\left(\rho_{\mathcal{S}}\right)$ about the state $\rho_{\mathcal{S}}$ of the system [here $S\left(\rho_{\mathcal{S}}\right)=-\operatorname{Tr}\left[\rho \log _{2} \rho\right]$ is the von Neumann entropy], the more widespread is the classical data recorded by the elements of the environment, which thus become an objective element of physical reality. Objectivity arises when many independent observers agree about a property of a system such as its state: independent observers looking at the same quantum system cannot have agreed on a particular measurement basis beforehand, and will generally measure different observables. The state of a closed quantum system cannot be seen as objective, in this respect: the measurements of noncommuting observables would contradict each other. Classically, instead, different observers can measure a system without affecting it. Therefore, classical states are objective by definition. The key observation of QD is that, when different fractions of an environment acquire the same (redundant) information over a single quantum system, the state of the latter must have become classical. The onset of such redundancy signals the occurrence of the quantum-to-classical transition.

Despite the key role that QD appears to play in the study of open-system dynamics, its phenomenology is yet to be fully characterized and it is in general interesting to determine its domain of validity [9]. The goal of this Rapid Communication is to experimentally address the effects of intraenvironment 
correlations in the occurrence of QD, thus addressing a situation deviating from the typical assumption of independent $\mathcal{E}_{j}$ 's typical of the QD framework.

We build our analysis on the use of tailored multiqubit graph states [10-14], within which we identify a system qubit and environmental ones. Graph states result from the evolution of an $N$-partite register of qubits initially prepared in $\otimes_{j=1}^{N} \hat{H}_{j}|+\rangle_{j}$, with $|+\rangle$ the eigenstate of the Pauli $\sigma_{x}$ operator with eigenvalue +1 , following the Hamiltonian of interaction

$$
\hat{H}=\frac{1}{4} \sum_{j, k=1}^{N} g_{j k}\left(\mathbb{1}-\sigma_{z}^{j}\right)\left(\mathbb{1}-\sigma_{z}^{k}\right) v,
$$

where $g_{j k}$ is a coupling rate (we assume units such that $\hbar=1$ ). The degree of correlations shared by any two qubits is a function of the rate $g_{j k}$. In our investigation we tune such parameters from $g_{j k}=0$, when the indices $j$ and $k$ pertain to environmental elements only, to the case of nonnull intraenvironment interactions. Therefore, we compare the case of independent subenvironments (in line with the QD assumptions) to that where strong intraenvironment correlations are set. This corresponds to different working regimes of an underlying dynamical model of which the graph states that we have considered are the result. The corresponding analysis of QD shows that significant deviations from the expectations for such phenomenon are in order in the latter case, with seemingly no redundant information about the state of $\mathcal{S}$ being recorded by the environment.

Brief description of $Q D$. Let us assume, for simplicity, that the initial state of the system reads $|\psi\rangle_{\mathcal{S}}=\sum_{k=1}^{n} \psi_{k}\left|s_{k}\right\rangle_{\mathcal{S}}$ with $\left\{\left|s_{k}\right\rangle_{\mathcal{S}}\right\}$ a basis in the Hilbert space of the $n$-dimensional system. The environment is prepared in $\left|\epsilon_{0}\right\rangle_{\mathcal{E}}=\otimes_{j=1}^{N}\left|\epsilon_{0}\right\rangle_{j}$ with $\left|\epsilon_{0}\right\rangle_{j}$ the initial state of the $j$ th subenvironment. The paradigm of QD requires the following typical evolution of the initial $\mathcal{S}-\mathcal{E}$ state:

$$
|\psi\rangle_{\mathcal{S}}\left|\epsilon_{0}\right\rangle_{\mathcal{E}} \longrightarrow \sum_{k=1}^{n} \psi_{k}\left|s_{k}\right\rangle_{\mathcal{S}}\left|\epsilon_{k}\right\rangle_{\mathcal{E}}
$$

with $\left|\epsilon_{k}\right\rangle_{\mathcal{E}}=\otimes_{j=1}^{N}\left|\epsilon_{k}\right\rangle_{j}$ the evolved state of the environment conditional on the system being in $\left|s_{k}\right\rangle_{\mathcal{S}}$. As all the subenvironments encode the same state and there is a strong correlation between $\mathcal{S}$ and $\mathcal{E}$, the information on the system is redundantly recorded into the environment (such information being the classical label $k$ that identifies the state component of the system).

The redundancy that is at the core of QD is well captured by the degree of total correlations set in the joint state of $\mathcal{S}$ and a fragment $\mathcal{F}$ of the environment, i.e., the set of subenvironments corresponding to a choice of the indices $j=1, \ldots, N$. Such total correlations are quantified by the mutual information

$$
I_{\mathcal{S F}}=H_{\mathcal{S}}+H_{\mathcal{F}}-H_{\mathcal{S}, \mathcal{F}} .
$$

The emergence of $\mathrm{QD}$, associated with the proliferation of information across $\mathcal{E}$, is marked by the insensitivity of $I_{\mathcal{S F}}$ to the dimension $\Delta(\mathcal{F})$ of the fragment $\mathcal{F}$ being considered. This happens when almost all of the information about $\mathcal{S}$ is contained in $\mathcal{F}$, so that $I_{\mathcal{S F}}$ quickly rises to $S_{\mathcal{S}}$, which is all of the available information about $\mathcal{S}$ (cf. Fig. 1). Equation (3) is the main instrument of the analysis that we present hereafter.

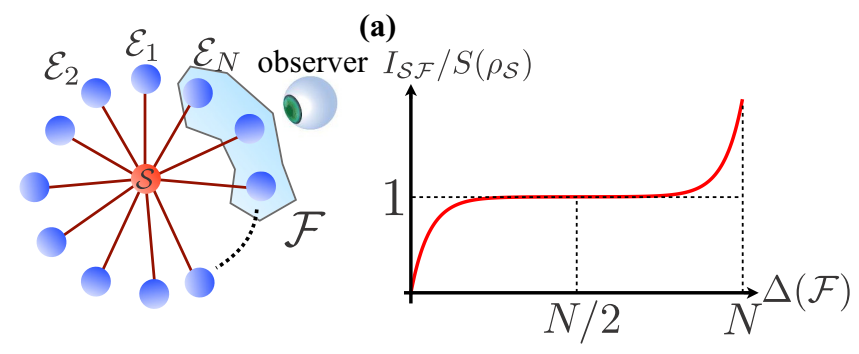

(b)

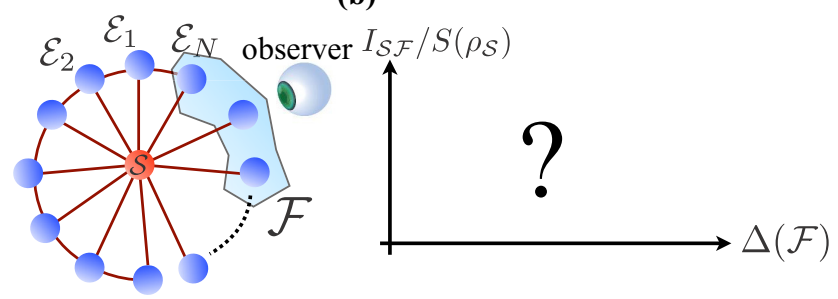

FIG. 1. Upper panel: under the assumptions of QD (independent subenvironments $\mathcal{E}_{j}$ ), the mutual information $I_{\mathcal{S F}}$ between the system $\mathcal{S}$ and a fragment $\mathcal{F}$ of the environment showcases a plateau against the size $\Delta(\mathcal{F})$ of the fragment itself, which witnesses the redundancy of the information encoded in the state of the elements of the environment. Lower panel: under the presence of intraenvironment correlations (indicated by the solid lines joining pairs of $\mathcal{E}_{j}$ particles), the phenomenology of QD is not known.

Resource state for the study of $Q D$ and its breakdown. Our analysis will be based on the use of graph states able to encode tunable correlations between the system and the environmental elements $\mathcal{E}_{j}$. Specifically, we consider states whose underlying graphs are akin to those in Fig. 2, and which we dub star-and diamond-shaped graph states [15]. Both can be synthesized from the general graph state of $N+1$ qubits

$$
\left|G_{N+1}\right\rangle=\prod_{j, k} \hat{C}\left(\phi_{j, k}\right)\left(\bigotimes_{l=1}^{N+1}|+\rangle_{l}\right),
$$

(a)

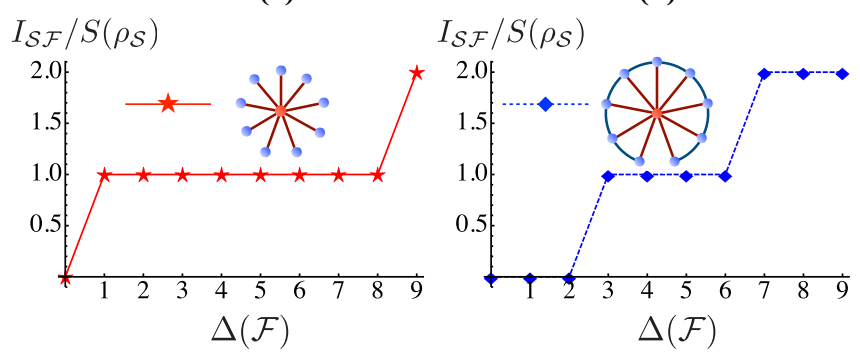

FIG. 2. Numerical analysis of the mutual information between the system and fragments of the environment as a function of the number of qubits in the fragment. The results are shown for a tenelement star-shaped [panel (a)] and diamond-shaped [panel (b)] graph state. We include the underlying graph of the states that have been considered in the calculations, which involve a system qubit $\mathcal{S}$ and nine elements of its environment $\mathcal{E}$. The burgundy-colored links are the interactions set between $\mathcal{S}$ and $\mathcal{E}_{j}$, while the blue-colored ones stand for the intraenvironment interactions. 

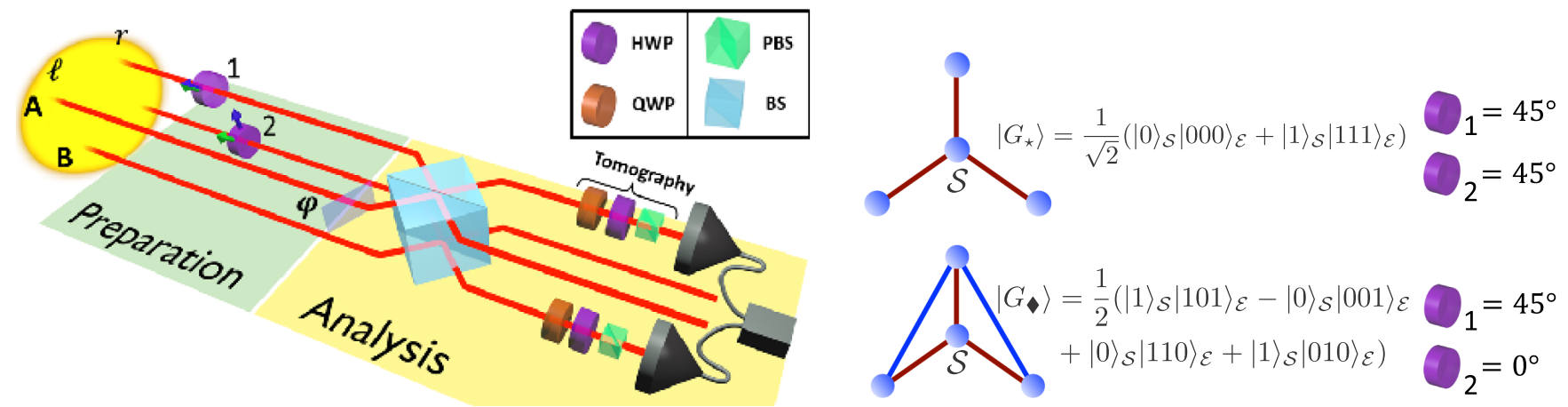

FIG. 3. Experimental scheme for the synthesis of diamond- and star-shaped graph states. In the figure, HWP stands for a half-wave plate, QWP is a quarter-wave plate, PBS is a polarizing beam splitter, and BS is a beam splitter. In the preparation stage, the resource states are built. The yellow halo represents the path-polarization two-photon four-qubit hyperentangled source described in Refs. [10,17]. Star-shaped graph state: the source generates the state $|H H\rangle_{A B} \otimes(|\ell r\rangle+|r \ell\rangle)_{A B}$; HWP1 and HWP2 are placed at $45^{\circ}$ on modes $r_{A}$ and $r_{B}$ (green arrows). Diamond-shaped graph state: the source generates $(|H H\rangle+|V V\rangle)_{A B} \otimes(|\ell r\rangle+|r \ell\rangle)_{A B}$; the half-wave plate HWP1 on mode $r_{A}$ is oriented at $45^{\circ}$, while HWP2 on mode $r_{B}$ is at $0^{\circ}$ (blue arrows). In the analysis stage, the path qubits are analyzed through a phase shifter $(\varphi)$ and a $\mathrm{BS}$, and the polarization qubits are analyzed through a standard tomographic setup. Interferometric filters select degenerate photons centered at $\lambda=710 \mathrm{~nm}$ with a $6 \mathrm{~nm}$ bandwidth, and coincidence counts between modes $r_{A}$ and $l_{B}$ are measured using single-photon counting modules in a time window of $\approx 9 \mathrm{~ns}$. Rates of $\approx 500$ coincidences/s are experimentally observed.

where we have introduced the controlled-phase gate $\hat{C}\left(\phi_{j, k}\right)$ $\left(\phi_{j, k}\right.$ is a real phase) acting on the qubit pair $(j, k)$ as

$$
\hat{C}\left(\phi_{j, k}\right)=|0\rangle\left\langle\left. 0\right|_{j} \otimes \mathbb{1}_{k}+\mid 1\right\rangle\left\langle\left. 1\right|_{j} \otimes\left(\begin{array}{cc}
1 & 0 \\
0 & e^{i \phi_{j, k}}
\end{array}\right)_{k} .\right.
$$

Such operation arises directly from the Hamiltonian in Eq. (1) for suitable choices of the coupling strength $g_{j k}$. Therefore, the states considered here represent the results of a dynamical model where the qubits under study evolve toward the special graph states in Fig. 2 . The choice of $\phi_{j, k}=\pi$ for any pair of qubits results in a standard cluster state [16].

We perform a controlled-phase gate with phase $\phi$ between the system qubit (which we assume to be qubit 1 for simplicity) and its neighboring qubits. On the other hand, we apply a controlled-phase gate with phase $\theta$ between the pairs of qubits of the environment. This results in

$$
\left|G_{N+1}\right\rangle=\prod_{j=2}^{N-2} \hat{C}\left(\theta_{j, j+1}\right) \prod_{k=2}^{N} \hat{C}\left(\phi_{\mathcal{S}, k}\right)\left(|+\rangle_{\mathcal{S}} \bigotimes_{l=2}^{N}|+\rangle_{l}\right)
$$

with $\phi_{\mathcal{S}, k}=\phi$ and $\theta_{j, j+1}=\theta$ for any choice of indices. By changing these values we change the strength of the correlations between the qubits. In particular, for $\phi=\pi$ and $\theta=0$, which correspond to the star-shaped graph state, no interaction among the elements of the environment is set, and thus no intraenvironment correlation. This corresponds to the standard assumptions in the QD picture. On the other hand, $\theta \neq 0$ corresponds to a diamond-shaped graph state of $\theta$-dependent degree of correlations among the $\mathcal{E}_{j}$ 's.

We analyze the mutual information shared between $\mathcal{S}$ and $\mathcal{E}$ as a function of fractions of the environment for such systems. The theoretical predictions are shown as the lines in Figs. 2(a) and 2(b). There is a strongly different behavior between the star- and the diamond-shaped graph state: the former has a constant mutual information, which is sign of occurrence of QD and the latter has a growing mutual information which denotes the disruption of QD. In light of the connection between the graph states considered here and the corresponding Hamiltonian model, these results should be seen as valid in a dynamical sense, where $\mathcal{S}$ and $\mathcal{E}$ interact continuously.

Experimental synthesis of star-and diamond-shaped graph states. We now illustrate the experimental procedure used to engineer representatives of the two classes of states discussed above (Fig. 3). Our approach is based on the use of a wellconsolidated and tested source of four-qubit path-polarization photonic hyperentangled cluster states [10,11].

A double-passage scheme through a nonlinear type-I $\beta$ barium-borate (BBO) crystal generates a two-photon state, entangled in polarization, while a four-hole mask, symmetrically placed over the center of the photons emission cone, generates entanglement between the paths of the photon pairs. The state generated by this source can be written as

$$
|\Xi\rangle=\frac{1}{\sqrt{2}}\left(|H H\rangle_{12}+|V V\rangle_{12}\right) \otimes \frac{1}{\sqrt{2}}\left(|\ell r\rangle_{34}+|r \ell\rangle_{34}\right) .
$$

Here $|H\rangle$ and $|V\rangle$ are horizontal and vertical polarization states of the two photons, respectively, while $\ell$ and $r$ are left and right paths of the photons through the mask. The label $j=1, \ldots, 4$ identifies the logical qubits at hand.

The full form of a four-qubit star-shaped graph state is

$$
\left|G_{\star}\right\rangle=\left[\prod_{j=2}^{4} \hat{C}\left(\phi_{\mathcal{S}, j}\right)\right]|++++\rangle_{1234},
$$

where $\phi_{\mathcal{S}, j}=\pi \forall j=2, \ldots, 4$. It can be seen that $\left(\otimes_{j=2}^{4} \hat{\mathcal{H}}_{j}\right)\left|G_{\star}\right\rangle=\left|\mathrm{GHZ}_{4}\right\rangle \quad$ with $\left|\mathrm{GHZ}_{4}\right\rangle=\left(|0000\rangle_{1234}+\right.$ $\left.|1111\rangle_{1234}\right) / \sqrt{2}$ a four-qubit Greenberger-Horne-Zeilinger (GHZ) state, and $\hat{\mathcal{H}}_{j}$ a Hadamard gate applied to qubit $j$. As local operation on single qubits does not change the mutual information within the state, we can equivalently analyze the GHZ state. This is retrieved from the experimental state in Eq. (7) by selecting only one of the cones of the emission from the BBO crystal and introducing two half-wave plates (HWPs) at $45^{\circ}$ on the paths of modes $r_{A}$ and $r_{B}$. The resulting 
state becomes $\left|G_{\star}\right\rangle_{\exp }=\frac{1}{\sqrt{2}}\left(|H V \ell r\rangle_{1234}+|V H r \ell\rangle_{1234}\right)$. The logical encoding $|H / V\rangle \rightarrow|0 / 1\rangle,|\ell / r\rangle \rightarrow|0 / 1\rangle$ gives us the state $(|0101\rangle+|1010\rangle) / \sqrt{2}$, which is locally equivalent to the GHZ state above.

As for the four-qubit diamond-shaped graph state, we can proceed as follows. The state is written as $\left|G_{\downarrow}\right\rangle=$ $\hat{C}\left(\theta_{23}\right) \hat{C}\left(\theta_{34}\right)\left|G_{\star}\right\rangle$ with $\theta_{23}=\theta_{34}=\pi$. A straightforward manipulation shows that

$$
\begin{aligned}
& \operatorname{Swap}_{2,3} \mathcal{H}_{1} \otimes\left(\sigma_{x} \mathcal{H}\right)_{2} \otimes \mathcal{H}_{3} \otimes\left(\sigma_{z} \mathcal{H}\right)_{4}\left|G_{\diamond}\right\rangle \\
& \quad=\frac{1}{2}(-|0001\rangle+|0110\rangle+|1010\rangle+|1101\rangle)_{1234}
\end{aligned}
$$

with $\mathrm{Swap}_{2,3}$ the SWAP gate applied to qubits 2 and 3. In order to build the state through our experimental setup, we start from Eq. (7) and we introduce a HWP at $45^{\circ}$ over mode $r_{A}$ and one at $0^{\circ}$ over mode $r_{B}$. The result is $\left|G_{\triangleleft}\right\rangle_{\exp }=\frac{1}{2}[-(|H H\rangle-$ $\left.|V V\rangle)_{12}|\ell r\rangle_{34}+(|H V\rangle+|V H\rangle)_{12}|r \ell\rangle_{34}\right]$, which is equivalent to Eq. (9).

Experimental assessment of $Q D$. A possible approach to study the mutual information between $\mathcal{S}$ and a growing-size environmental fragment $\mathcal{F}$ is the evaluation of $I_{\mathcal{S F}}$ over the reductions of the experimentally reconstructed $\mathcal{S}-\mathcal{E}$ density matrix. As a hyperentanglement-based approach allows for the fully independent control over the four qubits of our resource state, it represents an optimal platform for this assessment.

In Figs. 4(a)-4(d) we present the full state tomographies that have been experimentally determined to evaluate the quality of the generated states. We obtained a fidelity of $F_{\star}=$ $(91.0 \pm 0.7) \%$ for the star state, and $F_{\bullet}=(91 \pm 1) \%$ for the diamond state which highlights the good overall quality of our states.

However, a more direct estimation of the mutual information is possible adapting the methodology in Ref. [18], which is based on the decomposition of the mutual information between $\mathcal{S}$ and a given fragment $\mathcal{F}$ in terms of multiqubit correlators of the elements of the set of operators $\{\boldsymbol{\sigma}\}$ with $\sigma_{0}=\mathbb{1}$ and $\sigma_{1,2,3}$ the $x, y$, and $z$ Pauli matrix, respectively. In detail, let us define the four-point correlation functions evaluated over the density matrix $\rho_{\exp }^{k}(k=\star, \downarrow)$ describing the state of our four-qubit

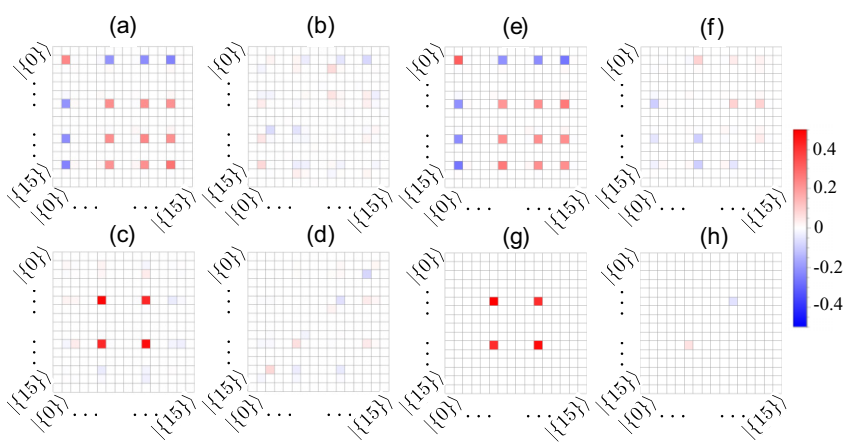

FIG. 4. Experimental density matrix of the diamond- (top) and star-shaped (bottom) graph states. Panels (a) and (c) [panels (b) and (d)] show the real (imaginary) parts of the density matrix. Panels (e) and (g) [panels (f) and (h)] show the real (imaginary) parts of the reconstructed density matrix entries. We have used the notation according to which $\{0\}=0000,\{1\}=0001, \ldots,\{15\}=1111$, according to a digital-to-binary encoding. resource

$$
\mathcal{C}_{\alpha \beta \gamma \delta}^{k}=\operatorname{Tr}\left[\rho^{k}\left(\sigma_{\alpha} \sigma_{\beta} \sigma_{\gamma} \sigma_{\delta}\right)\right](\alpha, \beta, \gamma, \delta=0, \ldots, 3),
$$

where we have omitted the symbol of tensor product for easiness of notation. Such correlators can be easily measured in our experimental setup, similarly to the procedure used to reconstruct the density matrix, by means of path projections, obtained by introducing a beam splitter (BS) over the four output modes of the mask [10], and polarization projections, obtained by a standard tomographic setup made by a quarterwave plate (QWP), a HWP, and a polarizing BS (PBS). Coincidence counts are measured by avalanche photodiodes in a gate of $\sim 9 \mathrm{~ns}$. Suitable combinations of such correlation functions allow us to reconstruct the element $\left(\rho^{k}\right)_{i j}$ of the density matrix as

$$
\left(\rho^{k}\right)_{i j}=\sum_{\alpha, \beta, \gamma, \delta=0}^{3} a_{\alpha \beta \gamma \delta}^{i j} \mathcal{C}_{\alpha \beta \gamma \delta}^{k} \quad(i, j=1,16)
$$

with $\left\{a_{\alpha \beta \gamma \delta}^{i j}\right\}$ as the set of (in general complex) numbers. The $\mathcal{S}-\mathcal{F}$ mutual information can then be cast as a function of such correlation functions. For instance, for the case of a star-shaped graph state we have $\rho^{\star}=\left|G_{\star}\right\rangle\left\langle G_{\star}|=P| 0101\right\rangle\langle 0101|+$ $(1-P)|1010\rangle\langle 1010|+(C|0101\rangle\langle 1010|+$ H.c. $)$

with

$$
\begin{aligned}
& P=\left[\mathcal{C}_{0000}+\mathcal{C}_{3333}-\mathcal{P}\left(\mathcal{C}_{0003}\right)-\mathcal{P}\left(\mathcal{C}_{0033}\right)+\mathcal{P}\left(\mathcal{C}_{0333}\right)\right] / 16, \\
& C=\left[\mathcal{C}_{1111}+\mathcal{C}_{2222}-i \mathcal{P}\left(\mathcal{C}_{1112}\right)-i \mathcal{P}\left(\mathcal{C}_{1222}\right)+\mathcal{P}\left(\mathcal{C}_{1122}\right)\right] / 16,
\end{aligned}
$$

where $\mathcal{P}\left(\mathcal{C}_{\alpha \beta \gamma \delta}\right)$ is the operator that performs the sum over the correlators obtained by permutation of the indices $\alpha, \beta, \gamma, \delta$. The mutual information $I_{\mathcal{S F}}^{\Delta(\mathcal{F})}$ for $\Delta(\mathcal{F})=1,2,3$ is then given by the following functions of $P$ and $C$ :

$$
\begin{aligned}
& I_{\mathcal{S F}}^{1}=I_{\mathcal{S F}}^{2}=-P_{r} \log \left(P_{r}\right)-(1-P)_{r} \log (1-P)_{r}, \\
& I_{\mathcal{S} \mathcal{F}}^{3}=\sum_{k= \pm} f_{r}^{k} \log f_{r}^{k}-2\left[P_{r} \log P_{r}+(1-P)_{r} \log (1-P)_{r}\right]
\end{aligned}
$$

with $f^{ \pm}=\left(2 P-1 \pm \sqrt{4|C|^{2}+(1-2 P)^{2}}\right) / 2$ and the subscript $r$ which stands for the real part of the corresponding function. Therefore, the experimental estimate of the mutual information can be performed by determining both $P$ and $C$ over the experimental state $\rho_{\exp }^{\star}$. This procedure requires the experimental evaluation of 32 correlators out of the 256 required for reconstructing the entire density matrix via a hypercomplete tomography. This method only requires 128 measurements, which compares favorably with the 1296 measurements that are required for a full quantum state tomography. A similar analysis can be performed for state $\rho_{\text {exp }}$. In this case, the density matrix has 16 expected nonzero elements, which can be reconstructed by a linear combination of 48 correlators. In Figs. 4(e)-4(h) we report the estimation of the density matrices obtained by following the procedure described above.

The experimental estimates of the mutual information for both the resource states are reported in Fig. 5, which demonstrates the striking differences in the behavior associated with the two configurations. The presence of correlations 


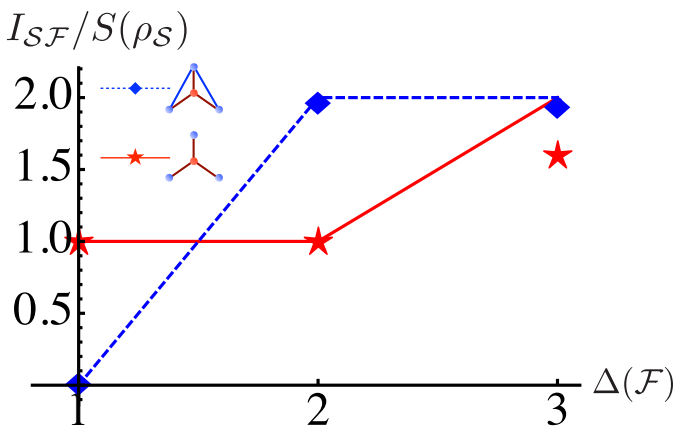

FIG. 5. Experimental mutual information between system and fractions of the environment, evaluated with the decomposition of the density matrix, as described in the main text. Red stars are for a star-shaped graph state, while the blue diamonds are for the diamond-shaped one. The red-solid and blue-dashed lines represent theoretical predictions. The error bars, representing a one-sigma confidence on the experimental point, are smaller than the dimensions of the symbols and are evaluated by considering Poissonian statistics on the photonic coincidence counts.

between elements of the environment, as is the case for a diamond-shaped graph state, results in an enhanced ability of the observers to gather information on the state of the system, which becomes maximum already when only $75 \%$ of the environmental elements is queried. Despite the small size of the resource, this feature emerges strikingly, de facto validating the expected trend of the mutual information in Fig. 2. A comment is in order: contrary to expectation and examples given in this respect [19], the observed breakdown of QD does not result from the emergence of non-Markovian dynamics due to the enforced interactions among the elements of the (finite-size) environment. In fact, the non-Markovian character of the system's dynamics in the diamond-shaped graph state is less pronounced than the one resulting from the star-shaped configuration. This suggests a more convoluted than anticipated relationship between memory-bearing environmental mechanisms and the emergence of objective reality. The establishment of such a link goes beyond the scope of this work and will be the focus of further investigations. Finally, we would like to mention that the discrepancy between the theoretical predictions and experimental observations for $\Delta(\mathcal{F})=3$ can be fully ascribed to the presence of unwanted elements in the experimental density matrices.

In order to support this claim in an intuitive, yet physically meaningful manner, we decided to follow a minimalistic approach, which is nevertheless quite informative. We have repeated the analysis of the mutual information between the system and a fraction of the environment by considering the state $\rho_{\text {model }}=\mathcal{N}\left(\rho_{\text {theory }}+\rho_{\text {unwanted }}\right)$ with $\rho_{\text {theory }}$ the density matrix corresponding to one of the ideal graph states and $\rho_{\text {unwanted }}$ a matrix with nonzero values-all being equal to the undetermined parameter $\alpha$-only in the unwanted entries of the corresponding experimental density matrix. Here, $\mathcal{N}$ is a suitable normalization factor. In Fig. 6 we report the results arising from such a model by showing the dependence of the

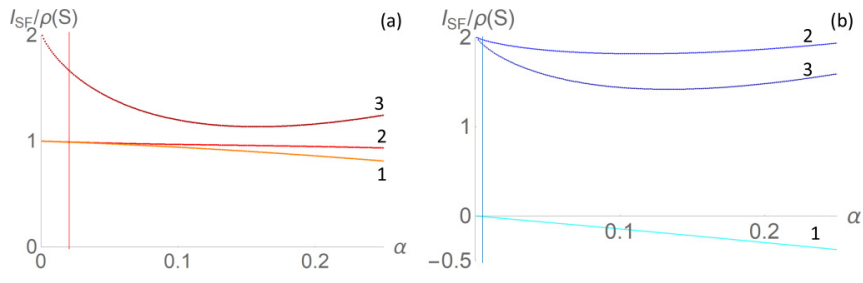

FIG. 6. Mutual information between system and environment as a function of the noise parameter $\alpha$, for the star cluster (a) and the diamond cluster (b). Each line is labeled by 1,2,3 and represents an environment made by $1,2,3$ qubits. The vertical line is the value of $\alpha$ such that the mutual information between system and three qubits of the environment is equivalent to the experimental result.

mutual information between the system and a growing number of environmental subsystems on the value taken by $\alpha$. We notice that, in the $\alpha \ll 1$ region where $\rho_{\text {model }}$ is physical and for both the star- and diamond-shaped graph states, the mutual information between $\mathcal{S}$ and an environmental fraction comprising only the first two elements of $\mathcal{E}$ is virtually unaffected by a nonzero value of $\alpha$. On the other hand, when we include the third environmental element, we observe a visible reduction in the mutual information as $\alpha$ grows. This is consistent with the behavior observed in Fig. 5 and provides an intuitive and simple justification of the discrepancies observed in that context. In particular, the curves corresponding to $\Delta(\mathcal{F})=3$ in Fig. 6 allow us to infer a value of $\alpha_{\star}=0.026 \pm 0.001$ and $\alpha \downarrow=0.003 \pm 0.001$ for the two graph states being considered, at which the corresponding mutual information matches the experimental values. Such estimates are fully plausible and in line with the values observed for the unwanted entries of the experimental density matrices.

Discussion. We have assessed the effect that strong intraenvironment correlations have over the emergence of QD in a controlled simulator of quantum open-system dynamics. We have shown that a simple graph configuration is able to encompass fundamental alterations, with respect to the behavior expected to arise from the typical QD paradigm [6-8], in the way information on the state of a quantum system is shared by the elements of an environment. Remarkably, the onset of such modifications to the QD phenomenology occurs already at a very small size (i.e., they are not emergent), which has enabled their experimental verification in a four-qubit photonic cluster state.

While representing a rare instance of an experimental case study on the emergence of objective reality (or the lack thereof), our work also highlights the complexity of this phenomenon, and its fragility with respect to the critical dependence of the actual system-environment dynamics from the prescriptions of QD.

Acknowledgments. This work was partially supported by the project FP7-ICT-2011-9-600838 (QWAD Quantum Waveguides Application and Development [20]). M.P. acknowledges the DfE-SFI Investigator Programme (Grant No. 15/IA/2864), the H2020 Collaborative Project TEQ (Grant No. 766900), and the Royal Society for financial support. 
[1] M. Schlosshauer, Rev. Mod. Phys. 76, 1267 (2004).

[2] W. H. Zurek, Rev. Mod. Phys. 75, 715 (2003).

[3] H.-P. Breuer and F. Petruccione, The Theory of Open Quantum Systems (Oxford University Press, New York, 2002).

[4] M. Schlosshauer, Decoherence and the Quantum-to-Classical Transition (Springer, Berlin/Heidelberg, 2007).

[5] A. Bassi, K. Lochan, S. Satin, T. P. Singh, and H. Ulbricht, Rev. Mod. Phys. 85, 471 (2013).

[6] W. H. Zurek, Phys. Today 44(10), 36 (1991).

[7] W. H. Zurek, S. Habib, and J. P. Paz, Phys. Rev. Lett. 70, 1187 (1993).

[8] W. H. Zurek, Nat. Phys. 5, 181 (2009).

[9] F. G. S. L. Brandao, M. Piani, and P. Horodecki, Nat. Commun. 6, 7908 (2015).

[10] M. Barbieri, C. Cinelli, P. Mataloni, and F. De Martini, Phys. Rev. A 72, 052110 (2005).

[11] C. Cinelli, M. Barbieri, R. Perris, P. Mataloni, and F. De Martini, Phys. Rev. Lett. 95, 240405 (2005).

[12] J. T. Barreiro, N. K. Langford, N. A. Peters, and P. G. Kwiat, Phys. Rev. Lett. 95, 260501 (2005).
[13] G. Vallone, E. Pomarico, P. Mataloni, F. De Martini, and V. Berardi, Phys. Rev. Lett. 98, 180502 (2007).

[14] K. Chen, C. M. Li, Q. Zhang, Y. A. Chen, A. Goebel, S. Chen, A. Mair, and J.-W. Pan, Phys. Rev. Lett. 99, 120503 (2007).

[15] A completely symmetric state resulting from the addition of a ring-shaped graph to the star-shaped one leads to the vanishing of quantum correlations in the environment. Therefore, such structure is equivalent to the star-shaped graph state, as far as QD is concerned.

[16] H. Briegel, D. E. Browne, W. Dür, R. Raussendorf, and M. Van den Nest, Nat. Phys. 5, 19 (2009).

[17] M. A. Ciampini, A. Orieux, S. Paesani, F. Sciarrino, G. Corrielli, A. Crespi, R. Ramponi, R. Osellame, and P. Mataloni, Light: Sci. Appl. 5, e16064 (2016).

[18] A. Chiuri, L. Mazzola, M. Paternostrostro, and P. Mataloni, New J. Phys. 14, 085006 (2012).

[19] F. Galve, S. Maniscalco, and R. Zambrini, Sci. Rep. 6, 19607 (2015).

[20] See www.qwad-project.eu. 